\title{
Anisotropic fluid with SU(2)-type structure in general relativity: A model of localized matter
}

\author{
Patricio S. Letelier \\ Departamento de Fisica Universidade de Brasília, 70910 Brasília, Brazil \\ Enric Verdaguer \\ Departament de Fisica Teòrica, Universitat Autònoma de Barcelona, Bellaterra (Barcelona), Spain
}

(Received 25 February 1987; accepted for publication 10 June 1987)

\begin{abstract}
A model of anisotropic fluid with three perfect fluid components in interaction is studied. Each fluid component obeys the stiff matter equation of state and is irrotational. The interaction is chosen to reproduce an integrable system of equations similar to the one associated to self-dual SU (2) gauge fields. An extension of the Belinsky-Zakharov version of the inverse scattering transform is presented and used to find soliton solutions to the coupled Einstein equations. A particular class of solutions that can be interpreted as lumps of matter propagating in empty space-time is examined.
\end{abstract}

\section{INTRODUCTION}

Anisotropic fluids are found in general relativity when electromagnetic fields or a viscous term are present. ${ }^{1}$ But they may also be found using two perfect fluid components ${ }^{2-4}$ or even more components. ${ }^{5}$

Models with multifluid components are increasingly being used in cosmology, ${ }^{6,7}$ in the description of collapsing spheres, ${ }^{8}$ and in the problem of halo and hole formation ${ }^{9,10}$ in expanding universes to represent inhomogeneous zones that develop galaxies and voids. "

In the present paper we study a model of anisotropic fluid with three perfect fluid components in interaction. Each fluid component obeys the stiff matter equation of state and is irrotational. The interaction is chosen to reproduce an integrable system of equations similar to the one associated to the Yang equations ${ }^{12}$ for self-dual SU(2) gauge fields with axial symmetry. For instance, these equations can be solved using a simple generalization of the Belinsky-Zakharov solution generating technique ${ }^{13}$ (BZSGT).

The application of the BZSGT opens the possibility of finding solitonlike solutions for the fluid. In particular, we can describe lumps of matter coupled to gravity propagating in empty space. The description of lumps is greatly simplified in the three-fluid model, since we only need a two-soliton solution, i.e., a scattering matrix with two complex poles. ${ }^{13}$ For the two-fluid model we need four complex poles, a fact that makes the analysis of the solutions quite complicated.

These solutions may be generalized to represent the collision of cylindrical lumps which may show some features of the merging of galaxies. These generalizations will be treated in a future paper.

In Sec. II we present a summary of the main formulas for the model of anisotropic fluid with multifluid components, of Ref. 5. In Sec. III we examine a three-fluid model with potentials interacting via a Yang-type of equation. In Sec. IV we study the Einstein equations coupled to the threefluid model for cylindrically symmetric space-times. In Sec. $V$ we present a class of two-soliton solutions for the self- gravitating anisotropic fluid of Sec. III and analyze a particular subclass that describes the propagation of a lump of matter on a flat space-time. In the Appendix we extend the usual BZSGT valid for symmetric matrices to the case of Hermitian matrices.

\section{A MODEL OF ANISOTROPIC FLUID WITH MULTIFLUID COMPONENTS}

The stress-energy tensor for the anistropic fluid is formed from the sum of three tensors, each of which is the energy-momentum tensor of a perfect fluid, in the particular case that the fluids' four-velocities are linearly dependent, ${ }^{5}$ i.e.,

$$
\begin{aligned}
& T^{\mu v}=\sum_{i=1}^{3} t_{(i)}^{\mu v}, \\
& t_{(i)}^{\mu v} \equiv\left(p_{i}+\rho_{i}\right) u_{(i)}^{\mu} u_{(i)}^{v}-p_{i} g^{\mu \nu} .
\end{aligned}
$$

The four-velocities $u_{(i)}^{\mu}$ are restricted by

$$
u_{(i)}^{\mu} u_{(i) \mu}=1 \text {, }
$$

and the existence of quantities $b_{i}$ different from 0 such that

$$
\sum_{i=1}^{3} b_{i} u_{(i)}^{\mu}=0 .
$$

The functions $\rho_{i}$ and $p_{i}$ are the fluids' rest energies and pressures, respectively.

With the transformations ${ }^{5}$

$$
\begin{aligned}
& \mu_{(1)}^{\mu} \rightarrow u_{(1)}^{* \mu}=\cos \phi u_{(1)}^{\mu}+\left(\frac{\beta+\gamma \epsilon_{12}}{\beta+\alpha \epsilon_{12}}\right)^{1 / 2} \sin \phi u_{(2)}^{\mu}, \\
& u_{(2)}^{\mu} \rightarrow u_{(2)}^{* \mu}=-\left(\frac{\beta+\alpha \epsilon_{12}}{\beta+\gamma \epsilon_{12}}\right)^{1 / 2} \sin \phi u_{(1)}^{\mu}+\cos \phi u_{(2)}^{\mu},
\end{aligned}
$$

where

$\tan (2 \phi)=2\left[\left(\beta+\alpha \epsilon_{12}\right)\left(\beta+\gamma \epsilon_{12}\right)\right]^{1 / 2} /(\alpha-\beta)$,

and the condition (2.4), we find that the energy-momentum tensor (2.1) can be cast in the form

$$
T^{\mu \nu}=(\rho+\pi) U^{\mu} U^{\nu}+(\sigma-\pi) \chi^{\mu} \chi^{\nu}-\pi g^{\mu \nu} .
$$


The quantities $\epsilon_{12}, \alpha, \gamma$, and $\beta$ are related to the fluid components by

$$
\begin{aligned}
& \epsilon_{i j}=\epsilon_{j i} \equiv u_{(i)}^{\mu} u_{(j) \mu}, \quad i, j=1,2,3, \\
& \alpha \equiv\left(p_{1}+\rho_{1}\right)+\left(p_{3}+\rho_{3}\right)\left(\frac{\epsilon_{13}-\epsilon_{23} \epsilon_{12}}{1-\epsilon_{12}^{2}}\right)^{2}, \\
& \gamma \equiv\left(p_{2}+\rho_{2}\right)+\left(p_{3}+\rho_{3}\right)\left(\frac{\epsilon_{23}-\epsilon_{12} \epsilon_{13}}{1-\epsilon_{12}^{2}}\right)^{2}, \\
& \beta \equiv\left(p_{3}+\rho_{3}\right) \frac{\left(\epsilon_{13}-\epsilon_{23} \epsilon_{12}\right)\left(\epsilon_{23}-\epsilon_{12} \epsilon_{13}\right)}{\left(1-\epsilon_{12}^{2}\right)^{2}} .
\end{aligned}
$$

The symbols $U^{\mu}, \chi^{\mu}, \rho, \sigma$, and $\pi$ represent the fluid flux velocity, the direction of anistropy, the fluid rest energy, the pressure along the anisotropy direction, and the pressure on the "perpendicular directions" to $\chi^{\mu}$, respectively. These quantities are related to the perfect fluid components by

$U^{\mu}=u_{(1)}^{* \mu} /\left(u_{(1)}^{* \alpha} u_{(1) \alpha}^{*}\right)^{1 / 2}$,

$\chi^{\mu}=u_{(2)}^{* \mu} /\left(-u_{(2)}^{* \alpha} u_{(2) \alpha}^{*}\right)^{1 / 2}$,

$\rho=\frac{1}{2}\left(\alpha+\gamma+2 \beta \epsilon_{12}-2 \pi\right)$

$$
+\frac{1}{2}\left[(\alpha-\gamma)^{2}+4\left(\beta+\alpha \epsilon_{12}\right)\left(\beta+\gamma \epsilon_{12}\right)\right]^{1 / 2},
$$

$\sigma=-\frac{1}{2}\left(\alpha+\gamma+2 \beta \epsilon_{12}-2 \pi\right)$

$$
+\frac{1}{2}\left[(\alpha-\gamma)^{2}+4\left(\beta+\alpha \epsilon_{12}\right)\left(\beta+\gamma \epsilon_{12}\right)\right]^{1 / 2},
$$

$\pi=p_{1}+p_{2}+p_{3}$.

Also, we have that

$$
\begin{aligned}
& U^{\mu} U_{\mu}=-\chi_{\mu} \chi^{\mu}=1, \quad U^{\mu} \chi_{\mu}=0, \\
& \rho=T^{\mu \nu} U_{\mu} U_{v}, \quad \sigma=T^{\mu \nu} \chi_{\mu} \chi_{\nu} .
\end{aligned}
$$

In general, it is necessary to add supplementary conditions to close the model; this point was treated in some detail in Ref. 2.

\section{A SPECIAL CASE OF MULTIFLUID WITH IRROTATIONAL COMPONENTS}

A simple closed model of fluid is obtained by assuming that each fluid four-velocity component is irrotational, i.e.,

$$
u_{(i)}^{\mu}=\phi_{(i)}^{\prime \mu} /\left(\phi_{(i), \alpha} \phi_{(i)}^{\prime \alpha}\right)^{1 / 2} \text {, }
$$

where, as usual, $\phi_{(i)}^{\prime \mu}=g^{\mu \alpha} \phi_{(i), \alpha}$ and $\phi_{(i), \alpha}=\partial_{\alpha} \phi_{(i)}$; and obeys the simple equation of state

$$
p_{i}=\rho_{i}=\frac{1}{2} \phi_{(1)}^{-2} \phi_{(i), \alpha} \phi_{(i)}^{\prime \alpha},
$$

i.e., each fluid obeys the stiff matter equation of state. Note that for the first component we recover the well-known Tabensky-Taub ${ }^{14}$ relations for the stiff fluid in terms of the potential $\Lambda \equiv \ln \phi_{(1)}$. Thus the multifluid with fluid components obeying (3.2) can be considered as the interaction of a Tabensky-Taub fluid with other two irrotational fluids. The case $\phi_{(3)}=0$ corresponds to the fluid studied in Ref. 4 .

The condition of linear dependence of the fluids' fourvelocities in this case reads

$$
\mathbf{b} \cdot \phi^{\mu}=0,
$$

where we have introduced the notation

$$
\mathbf{A} \cdot \mathbf{B}=\sum_{i=1}^{3} A_{(i)} B_{(i)} .
$$

From (2.1), (3.1), and (3.2) we find

$$
T_{\mu \nu}=\phi_{(1)}^{-2}\left(\phi_{, \mu} \cdot \phi_{, \nu}-\frac{1}{2} g_{\mu \nu} \phi_{, \alpha} \cdot \phi^{, \alpha}\right) \text {. }
$$

The energy-momentum tensor (3.5) can also be derived, in the usual way, from the Lagrangian density

$$
\mathscr{L}=\frac{1}{2} \sqrt{-g} \phi_{(1)}^{-2} \phi_{, \alpha} \cdot \phi^{\prime \alpha} \text {. }
$$

The simpler condition to determine the fields $\phi_{(i)}$ is to impose that they satisfy the Euler-Lagrange equations obtained from (3.6), i.e.,

$$
\begin{aligned}
& \left(\sqrt{-g} g^{\alpha \beta} \phi_{(1)}^{-2} \phi_{(1), \beta}\right)_{, \alpha}+\sqrt{-g} \phi_{(1)}^{-3} \phi_{, \alpha} \cdot \phi^{\alpha}=0, \\
& \left(\sqrt{-g} g^{\alpha \beta} \phi_{(1)}^{-2} \phi_{(2), \beta}\right)_{, \alpha}=0, \\
& \left(\sqrt{-g} g^{\alpha \beta} \phi_{(1)}^{-2} \phi_{(3), \beta}\right)_{, \alpha}=0 .
\end{aligned}
$$

The energy-momentum tensor (3.5) obeys

$$
\nabla_{\mu} T^{\mu \nu}=0
$$

as a consequence of (3.7). And for each fluid component we have

$$
\nabla_{\mu} t_{(i)}^{\mu v} \neq 0 .
$$

The relations (3.8) and (3.9) tell us that the whole fluid is a closed system with "internal" fluid components in interaction. Also, the anisotropic fluid is completely determinated by the fields $\phi_{(i)}$ and their evolution equations (3.7), i.e., no other extra equation like an equation of state is needed. As a matter of fact, the anisotropic fluid is completely determined by the quantities $\alpha, \beta, \gamma, \pi$, and $\epsilon_{12}$ that in terms of $\phi_{(i)}$ can be written as

$$
\begin{aligned}
& \pi=\left(1 / 2 \phi_{(1)}^{2}\right) \phi_{, \mu} \cdot \phi^{\mu}, \\
& \epsilon_{i j}=\lambda_{i j} / \sqrt{\lambda_{i i} \lambda_{j i}},
\end{aligned}
$$$$
\alpha=\frac{\lambda_{11}}{\phi_{(1)}^{2}}\left[1+\left(\frac{\lambda_{13} \lambda_{22}-\lambda_{12} \lambda_{23}}{\lambda_{11} \lambda_{22}-\lambda_{12}^{2}}\right)^{2}\right],
$$$$
\gamma=\frac{\lambda_{22}}{\phi_{(1)}^{2}}\left[1+\left(\frac{\lambda_{11} \lambda_{23}-\lambda_{12} \lambda_{13}}{\lambda_{11} \lambda_{22}-\lambda_{12}^{2}}\right)^{2}\right] \text {, }
$$$$
\beta=\sqrt{\lambda_{11} \lambda_{22}} \frac{\left(\lambda_{22} \lambda_{13}-\lambda_{12} \lambda_{23}\right)\left(\lambda_{11} \lambda_{23}-\lambda_{12} \lambda_{13}\right)}{\phi_{(1)}^{2}\left(\lambda_{11} \lambda_{22}-\lambda_{12}^{2}\right)^{2}},
$$

where

$$
\lambda_{i j}=\phi_{(i)}{ }^{\mu} \phi_{(j), \mu} .
$$

\section{EINSTEIN EQUATIONS COUPLED TO MATTER}

The Einstein equations

$$
R_{\mu \nu}-\frac{1}{2} g_{\mu \nu} R=-T_{\mu \nu}
$$

coupled to the energy-momentum tensor (3.5) are equivalent to

$$
\boldsymbol{R}_{\mu \nu}=-\phi_{(1)}^{-2} \boldsymbol{\phi}_{, \mu} \cdot \boldsymbol{\phi}_{, v} .
$$

The integrability of (4.1) is guaranteed by the field equations (3.7).

We shall consider a space-time with the cylindrically symmetric metric

$$
d s^{2}=e^{\omega}\left(d t^{2}-d r^{2}\right)-\gamma_{a b} d x^{a} d x^{b},
$$

where the sum convention is assumed in the indices $a$ and $b$ 
that take the values 2 and $3 ;\left(x^{0}, x^{1}, x^{2}, x^{3}\right)=(t, r, \theta, z), \gamma_{a b}$ and $\omega$ are functions of $t$ and $r$ only, and

$$
\begin{aligned}
& \gamma \equiv\left(\gamma_{a b}\right)=\gamma^{T}, \\
& \operatorname{det} \gamma=t^{2} .
\end{aligned}
$$

From (4.2)-(4.5) and the fact that cylindrical symmetry implies that the $\phi_{(i)}$ are functions of $t$ and $r$ only, we get

$$
\begin{aligned}
& -\left(R_{00}+R_{11}\right) \\
& =\omega_{, t} / t+1 / t^{2}+\frac{1}{4} \operatorname{tr}\left(\gamma_{, t} \gamma_{, t}^{-1}+\gamma_{, r} \gamma_{, r}^{-1}\right), \\
& =\phi_{(1)}^{-2}\left(\phi_{, t} \cdot \phi_{, t}+\phi_{, r} \cdot \phi_{, r}\right), \\
& -2 R_{01}=\omega_{, r} / t+\frac{1}{2} \operatorname{tr}\left(\gamma_{, t} \gamma_{, r}^{-1}\right), \\
& =2 \phi_{(1)}^{-2} \phi_{, t} \cdot \phi_{, r},
\end{aligned}
$$

and

$$
\left(t \gamma_{, t} \gamma^{-1}\right)_{, t}-\left(t \gamma_{, r} \gamma^{-1}\right)_{, r}=0
$$

where $\gamma^{-1}{ }_{, \mu} \equiv\left(\gamma^{-1}\right)_{, \mu}$.

The field equations (3.7) in space-time with the metric (4.3) reduce to

$$
\begin{aligned}
& \phi_{(1), t t}+\phi_{(1), t} / t-\phi_{(1), r r}+\phi_{(1)}^{-1}\left(\phi_{(2), t}^{2}-\phi_{(2), r}^{2}+\phi_{(3), t}^{2}\right. \\
& \left.-\phi_{(3), r}^{2}-\phi_{(1), t}^{2}+\phi_{(1), r}^{2}\right)=0 \\
& \left(t \phi_{(1)}^{-2} \phi_{(2), t}\right)_{, t}-\left(t \phi_{(1)}^{-2} \phi_{(2), r}\right)_{, r}=0 \\
& \left(t \phi_{(1)}^{-2} \phi_{(3), t}\right)_{, t}-\left(t \phi_{(1)}^{-2} \phi_{(3), r}\right)_{, r}=0
\end{aligned}
$$

These three equations can be written in a completely equivalent form as the single matrix equation

$$
\left(t Q_{, t} Q^{-1}\right)_{, t}-\left(t Q_{, r} Q^{-1}\right)_{, r}=0,
$$

where

$$
Q \equiv \frac{t}{\phi_{(1)}}\left(\begin{array}{cc}
1 & \phi_{(2)}-i \phi_{(3)} \\
\phi_{(2)}+i \phi_{(3)} & \phi \cdot \phi
\end{array}\right) .
$$

Note that

$$
\begin{aligned}
& Q=Q^{\dagger}, \\
& \operatorname{det} Q=t^{2}
\end{aligned}
$$

By using definition (4.11) it is not difficult to prove the following useful identities:

$$
\begin{aligned}
& \operatorname{tr}\left(Q_{, r} Q_{, r}^{-1}\right)=-2 \phi_{(1)}^{-2} \phi_{, r} \cdot \phi_{, r}, \\
& \operatorname{tr}\left(Q_{, t} Q_{, t}^{-1}\right)=-2\left(t^{-2}+\phi_{(1)}^{-2} \phi_{, t} \cdot \phi_{, t}\right), \\
& \operatorname{tr}\left(Q_{, r} Q_{, t}^{-1}\right)=-2 \phi_{(1)}^{-2} \phi_{, t} \cdot \phi_{, r} .
\end{aligned}
$$

From (4.6), (4.7), and (4.14) we get

$$
\begin{aligned}
\omega_{, t}= & -2 / t-(t / 4) \operatorname{tr}\left(\gamma_{, t} \gamma_{, t}^{-1}+\gamma_{, r} \gamma_{, r}^{-1}\right) \\
& -(t / 2) \operatorname{tr}\left(Q_{, r} Q_{, r}^{-1}+Q_{, t} Q_{, t}^{-1}\right), \\
\omega_{, r}= & -(t / 2) \operatorname{tr}\left(\gamma_{, t} \gamma_{, r}^{-1}\right)-t \operatorname{tr}\left(Q_{, t} Q_{, r}^{-1}\right) .
\end{aligned}
$$

The existence of $\omega$, i.e., $\omega_{, t r}=\omega_{, r t}$, is a direct consequence of Eqs. (4.8) and (4.10). Thus the solution of the Einstein equations (4.2) for the metric (4.3) reduces to the solution of (4.8) and (4.10), and the computation of a quadrature for the coefficient $\omega$. [Compare Eqs. (4.15).]

In the case under consideration we have that the condition (3.3) is automatically satisfied as a consequence of the dependence of the function $\phi_{(i)}$ in only two variables, $t$ and $r$. Then, we can have the anisotropic fluid interpretation of the field equations (4.2). We find, after some algebra, that the quantities that appear in (2.8) - (2.12) can be written as

$$
\begin{aligned}
& \rho=\sigma=\frac{1}{2} \phi_{(1)}^{-2} e^{-\omega}\left|\phi_{, t}-\phi_{, r}\right|\left|\phi_{, t}+\phi_{, r}\right|, \\
& \pi=\frac{1}{2} \phi_{(1)}^{-2} e^{-\omega}\left(\phi_{, t} \cdot \phi_{, t}-\phi_{, r} \cdot \phi_{, r}\right), \\
& U^{\mu}=\frac{e^{-\omega / 2}}{\sqrt{2 \theta_{0}}}\left[\frac{2 \theta_{2}}{\left(\theta_{1}-\theta_{0}\right)^{1 / 2}},-\left(\theta_{1}-\theta_{0}\right)^{1 / 2}, 0,0\right], \\
& \chi^{\mu}=\frac{e^{-\omega / 2}}{\sqrt{2 \theta_{0}}}\left[\frac{2 \theta_{2}}{\left(\theta_{1}+\theta_{0}\right)^{1 / 2}},-\left(\theta_{1}+\theta_{0}\right)^{1 / 2}, 0,0\right],
\end{aligned}
$$

where the vertical bars indicate the usual Euclidean norm and

$$
\begin{aligned}
& \theta_{0}=\left|\phi_{, t}-\phi_{, r}\right|\left|\phi_{, t}+\phi_{, r}\right|, \\
& \theta_{1}=\phi_{, t} \cdot \phi_{, t}+\phi_{, r} \cdot \phi_{, r}, \\
& \theta_{2}=\phi_{, t} \cdot \phi_{, r} .
\end{aligned}
$$

Two useful identities are

$$
\begin{aligned}
& \alpha+\gamma+2 \beta \epsilon_{12}-2 \pi=0, \\
& \theta_{0}^{2}+4 \theta_{2}^{2}-\theta_{1}^{2}=0 .
\end{aligned}
$$

Equation (4.21) is a consequence of (3.10)-(3.15) and (4.3), and (4.22) follows from (4.20).

$$
\begin{aligned}
& \text { Also, we have } \\
& \phi_{(1)}=t / Q_{11}, \\
& \phi_{(2)}=\operatorname{Re}\left(Q_{12}\right) / Q_{11}, \\
& \phi_{(3)}=-\operatorname{Im}\left(Q_{12}\right) / Q_{11} .
\end{aligned}
$$

Expressions (4.16)-(4.19) can also be obtained directly by solving the eigenvalue problem for the tensor (3.5) with the metric (4.3) and $\phi_{(i)}$ functions of $t$ and $r$ only. In other words, the anisotropic fluid interpretation of (4.3) is independent of the identifications (3.1) and (3.2). Thus, even though (3.1) is meaningless in the case that $\phi_{(i), \alpha} \phi_{(i)}{ }^{, \alpha}<0$ the anisotropic fluid interpretation of (4.3) is still valid. The only problem that one has is that $\pi<0$. Note that the same problem occurs in the one-fluid case (Tabensky-Taub fluid). ${ }^{14,15}$

Equations (3.7) can also be cast as a matrix equation in terms of the matrix $Q$ whose elements are space-time scalars [cf. Eq. (4.11)],

$$
\nabla^{\mu}\left(Q_{, \mu} Q^{-1}\right)=0 \text {. }
$$

In the case of Euclidean metric $g_{\mu v}=\delta_{\mu v}$, Eq. (4.24) is closely related to the equation for self-dual $\mathrm{SU}(2)$ gauge fields in the Yang gauge. ${ }^{12}$ For a cylindrically symmetric Euclidean space-time (4.24) reduces exactly to the Yang equation for axially symmetric instantons. ${ }^{16}$ Hence (4.10) can be considered as the hyperbolic version of the Yang equation for self-dual $\mathrm{SU}(2)$ gauge fields.

The real case, i.e., $Q=Q^{r}$ is equivalent to the case studied in Ref. 4. Note that in this case we also have $\rho=\sigma$, i.e., a stiff equation of state in the direction of anisotropy.

\section{PARTICULAR SOLITARY WAVE SOLUTION}

There are many different techniques used to solve Eq. (4.10), the most commonly used are the Bäcklund transformations and the inverse scattering method. In this section we study a particular solution obtained using an extension of the 
Belinsky-Zakharov ${ }^{13}$ version of the inverse scattering transform that we present in the Appendix.

We shall focus our attention mainly in the matter content of the solution, for this reason we take the metric as being diagonal along the complete evolution of the spacetime, i.e.,

$$
\gamma_{0}=t\left(\begin{array}{cc}
e^{\Omega} & 0 \\
0 & e^{-\Omega}
\end{array}\right)
$$

The solution for the matter is generated using the BelinskyZakharov solution generating technique with the diagonal seed solution

$$
Q_{0}=t\left(\begin{array}{cc}
e^{-\Lambda} & 0 \\
0 & e^{\wedge}
\end{array}\right)
$$

In this case we have that the Einstein equations (4.8), (4.10), and (4.15) and the metric (4.3) can be written as

$$
\begin{aligned}
& d s^{2}=e^{\omega_{00}}\left(d t^{2}-d r^{2}\right)-t\left(e^{\Omega} d \theta^{2}+e^{-\Omega} d z^{2}\right), \\
& \omega_{00}=-\frac{1}{2} \ln t+v[\Omega]+2 v[\Lambda], \\
& v[\Omega] \equiv \frac{1}{2} \int t\left[\left(\Omega_{, t}^{2}+\Omega_{, r}^{2}\right) d t+2 \Omega_{, t} \Omega_{, r} d r\right], \\
& \Omega_{, t t}+\Omega_{, t} / t-\Omega_{, r r}=0, \\
& \Lambda_{, t t}+\Lambda_{, t} / t-\Lambda_{, r r}=0 .
\end{aligned}
$$

This particular solution to the Einstein equation for a single fluid component, i.e.,

$$
\begin{aligned}
& \phi_{(1)}=e^{\Lambda}, \\
& \phi_{(2)}=\phi_{(3)}=0,
\end{aligned}
$$

obeying the stiff equation of motion $p_{1}=\rho_{1}$ is studied in Refs. 15 and 17. Note that one recovers the vacuum solution (Einstein-Rosen ${ }^{18}$ solution) in the case $\Lambda=0\left(\phi_{(1)}=1\right)$ and $\phi_{(2)}=\phi_{(3)}=0$.

The application of the one-soluton BZSGT to the seed solution (5.2), i.e., to the matter only, yields the solution (see the Appendix)

$\omega_{01}=\omega_{00}+2 \ln \frac{t^{1 / 2}\left|\mu_{1}\right|\left(\left|p_{1}\right|^{2}\left|Y_{1}\right|^{2}+\left|q_{1}\right|^{2}\left|Y_{1}\right|^{-2}\right)}{\left[\left(\left|\mu_{1}\right|^{2}-t^{2}\right)\left|\mu_{1}^{2}-t^{2}\right|\right]^{1 / 2}}$,

$\phi_{(1)}=e^{\Lambda} \frac{\left|p_{1}\right|^{2}\left|Y_{1}\right|^{2}+\left|q_{1}\right|^{2}\left|Y_{1}\right|^{-2}}{\left|p_{1}\right|^{2}\left|Y_{1} / X_{1}\right|+\left|q_{1}\right|^{2}\left|Y_{1} / X_{1}\right|^{-2}}$,

$\phi_{(2)}=-e^{\wedge} \frac{\left(\left|X_{1}\right|^{2}-\left|X_{1}\right|^{-2}\right) \operatorname{Re}\left(\bar{p}_{1} q_{1} \bar{Y}_{1} / Y_{1}\right)}{\left|p_{1}\right|^{2}\left|Y_{1} / X_{1}\right|^{2}+\left|q_{1}\right|^{2}\left|Y_{1} / X_{1}\right|^{-2}}$,

$\phi_{(3)}=e^{\wedge} \frac{\left(\left|X_{1}\right|^{2}-\left|X_{1}\right|^{-2}\right) \operatorname{Im}\left(\bar{p}_{1} q_{1} \bar{Y}_{1} / Y_{1}\right)}{\left|p_{1}\right|^{2}\left|Y_{1} / X_{1}\right|^{2}+\left|q_{1}\right|^{2}\left|Y_{1} / X_{1}\right|^{-2}}$,

where

$$
\begin{aligned}
& Y_{k} \equiv \exp \left(F_{k}-\Lambda / 2\right) \\
& X_{k} \equiv\left(\mu_{k} / t\right)^{1 / 2}
\end{aligned}
$$
by

By doing $\Lambda=q_{1}=0$ we have the solution characterized

$$
\begin{aligned}
& \omega_{01}=\frac{1}{2} \ln t+v[\Omega]+\ln \frac{\left|\mu_{1}-\bar{\mu}_{1}\right|}{\left|\mu_{1}^{2}-t^{2}\right|} \\
& \phi_{(1)}=\left|\mu_{1} / t\right| \\
& \phi_{(2)}=\phi_{(3)}=0 .
\end{aligned}
$$

To derive (5.13) we have assumed that $\operatorname{Im} \mu_{1} \neq 0$ used the identity

$$
\left(\mu_{2}-\mu_{1}\right)\left(\mu_{1} \mu_{2}-t^{2}\right)=2\left(\alpha_{2}-\alpha_{1}\right) \mu_{1} \mu_{2},
$$

and disregarded a constant of integration, a practice that we shall follow without warning.

A more interesting solution is obtained by applying the same one-soliton BZSGT to the diagonal one-soliton already found. We find the two-soliton solution

$$
\begin{aligned}
\omega_{02}= & \frac{3}{2} \ln t+v[\Omega] 1+\ln \frac{\left|\mu_{1}-\bar{\mu}_{1}\right|\left|\mu_{2}-\bar{\mu}_{2}\right|}{\left|\mu_{1}^{2}-t^{2}\right|\left|\mu_{2}^{2}-t^{2}\right|} \\
& +2 \ln \left(\left|p_{2}\right|^{2}\left|Y_{2}\right|^{2}+\left|q_{2}\right|^{2}\left|Y_{2}\right|^{-2}\right), \\
\phi_{(1)}= & \frac{\left|\mu_{1}\right|}{t} \frac{\left|p_{2}\right|^{2}\left|Y_{2}\right|^{2}+\left|q_{2}\right|^{2}\left|Y_{2}\right|^{-2}}{\left|p_{2}\right|^{2}\left|Y_{2} / X_{2}\right|^{2}+\left|q_{2}\right|\left|Y_{2} / X_{2}\right|^{-2}}, \\
\phi_{(2)}= & -\frac{\left|\mu_{1}\right|}{t} \frac{\left(\left|X_{2}\right|^{2}-\left|X_{2}\right|^{-2}\right) \operatorname{Re}\left(\bar{p}_{2} q_{2} \bar{Y}_{2} / Y_{2}\right)}{\left|p_{2}\right|^{2}\left|Y_{2} / X_{2}\right|^{2}+\left|q_{2}\right|^{2}\left|Y_{2} / X_{2}\right|^{-2}}, \\
\phi_{(3)}= & \frac{\left|\mu_{1}\right|}{t} \frac{\left(\left|X_{2}\right|^{2}-\left|X_{2}\right|^{-2}\right) \operatorname{Im}\left(\bar{p}_{2} q_{2} \bar{Y}_{2} / Y_{2}\right)}{\left|p_{2}\right|^{2}\left|Y_{2} / X_{2}\right|^{2}+\left|q_{2}\right|^{2}\left|Y_{2} / X_{2}\right|^{-2}},
\end{aligned}
$$

where

$$
Y_{2}=\left(\frac{t\left(\mu_{1}-\mu_{2}\right)\left(\bar{\mu}_{1}-\mu_{2}\right)}{\mu_{2}\left|\mu_{1}\right|}\right)^{1 / 2},
$$

and $X_{2}$ is given by (5.12).

The two-soliton solution (5.16) and (5.17) is a particular case of the complete two-soliton obtained from the vacuum as seed solution.

The solution can be used to represent localized distributions of matter with cylindrical symmetry propagating in empty space. This is not possible with the one-soliton solution (5.13) and (5.14) because the fluid potential $\phi_{(1)} \mathrm{di}$ verges when $r \rightarrow \infty$.

From the application of the BZSGT to some cosmological solutions we know ${ }^{19}$ that two independent complex pole trajectories are needed in order to obtain localized solutions (gravitational solitons). Moreover since the metric coefficients have to be real, for each complex pole its complex conjugate is also a pole ${ }^{13}$; so that we need four pole trajectories in all. However in the present case, since the matrix $Q$ describing the fluid potentials is not real, localized solutions can be obtained by using two complex pole trajectories only.

The way by which localized solutions are obtained is by taking opposite signs in the square roots of the two pole trajectories $\mu_{1}$ and $\mu_{2}$ [see Eq. (A6)]. With such a prescription it is easy to see that the fluid potentials $\phi_{(i)}(i=1,2,3)$, from (5.17), approach the seed values $\left(\phi_{(1)}=1, \phi_{(2)}=\phi_{(3)}\right.$ $=0$ ) in the asymptotic regions in the following way. They approach the seed decreasing like $r^{-1}$ at $t \ll r \rightarrow \infty$, they decrease like $t^{-1}$ at $r \ll t \rightarrow \infty$, and decrease like $t^{-1 / 2}$ along the light cone $r \sim t \rightarrow \infty$ This behavior is typical of the gravitational solitons in cosmological ${ }^{20-23}$ or cylindrical models ${ }^{24}$ and is an indication that the anistropic fluid is localized around the light cone $r=t$.

In Fig. 1 the fluid density $\rho$ and pressure along the radial direction $\sigma(=\rho)$ of Eq. (4.16) is represented for the fluid potentials (5.17). We take a negative sign for the square root of $\mu_{1}$ and a positive sign for that of $\mu_{2}$. The density is mainly localized around a cylinder that expands at the speed of 


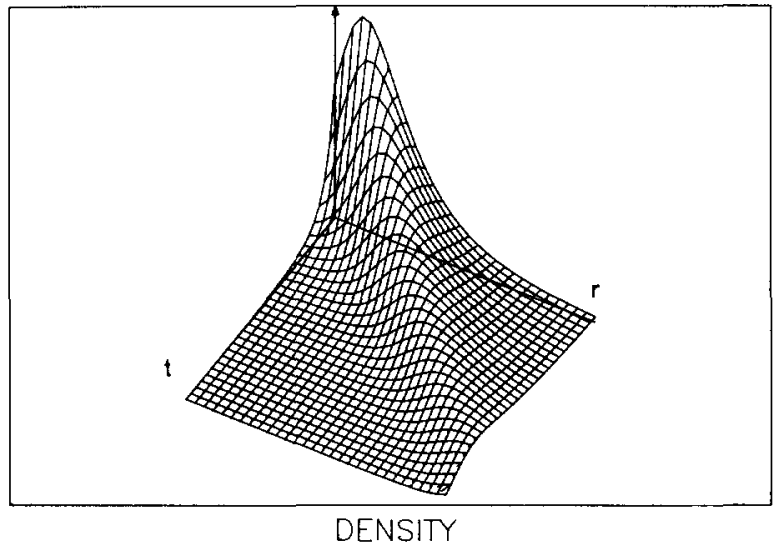

FIG. 1. Density $\rho$ and pressure along the radial direction are given in Eq. (4.16) with the fluid potentials (5.17). The parameters taken are $p_{2}=q_{2}=1$ and $\alpha_{1}=-0.2 i, \alpha_{2}=-0.1 i$. The time and radial coordinates, $t$ and $r$, are both represented in the range $(0.1,1)$. The relatively large value of $\left|\alpha_{1}-\alpha_{2}\right|$ has been taken to avoid sharp picks and to obtain a smooth figure. The background outside the wave is $\rho=\sigma \sim 0$, the spacetime axes are drawn on the origin of the vertical axis: $\rho=0$. The maximum value of $\rho$ is 7.5 .

light. The shape of the wave (its amplitude and width) is controlled by the imaginary part of $\alpha_{1}-\alpha_{2}$. The amplitude of the density wave decreases as the wave gets far from the origin. The wave propagates on an essentially empty background $(\rho=0)$.

In Fig. 2 the pressure $\pi$, Eq. (4.17), tangent to the cylinder is shown. As for the density we have a wave essentially localized along the light cone which propagates on an empty background $(\pi=0)$. The peculiarity here is that $\pi$ takes negative values on the region $r \gtrsim t$. As mentioned in Sec. IV the interpretation in terms of the fluid (3.1) is not possible although a fluid interpretation is still valid (see Ref. 15).

This model can be used to represent lumps of matter coupled to gravity propagating on empty space. The qualitative similarity of the waves of matter with the gravitational solitons, ${ }^{20,21}$ which are similar to the hydrodynamical solitons, suggests that the collision of lumps of matter might also

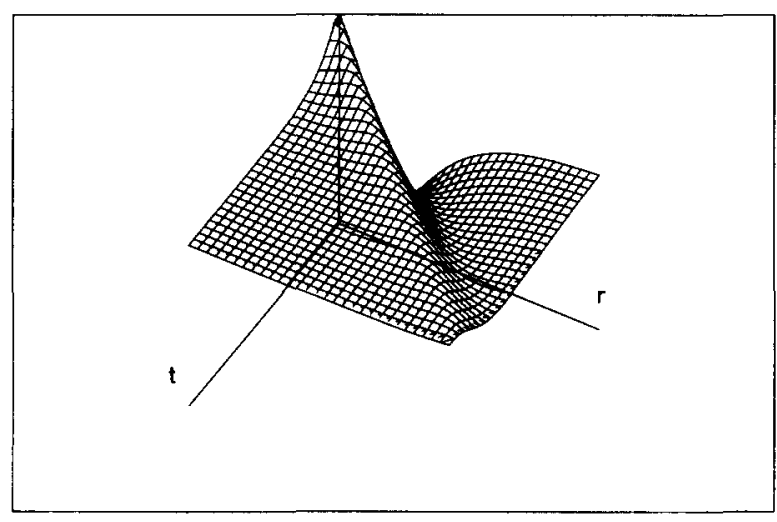

PRESSURE

FIG. 2. Pressure $\pi$ is tangent to the cylinder of Eq. (4.17), with the same parameters as in Fig. 1. The pressure takes positive and negative values in different regions of the space-time. The background value outside the "perturbation" is $\pi \sim 0$; the space-time axes are drawn in the negative region of the vertical axis: $\pi=-5$. have a solitonlike character. Models representing such collisions are being considered by the authors.

A word should be said about the equality $\rho=\sigma$. Although this is not verified by the general three-fluid case (2.12) $-(2.18)$ it is a feature of the cylindrical case, already present in the two-fluid case. ${ }^{4}$ The stiff equation of state verified along the propagation direction seems to be a feature of systems which admit solitons. ${ }^{22}$ The reason may be traced to the fact that there is a unique velocity on the system in the direction of propagation of the wave, ${ }^{24}$ in this case the speed of sound and the speed of the gravitational field are the same: It is known, for instance, ${ }^{25}$ that an initial perturbation with cylindrical symmetry on a perfect fluid coupled to gravity will disperse and form shock waves unless the fluid is stiff.

\section{ACKNOWLEDGMENTS}

We want to thank the following institutions for financial support: "Comisión Asesora para la Investigación de España" (EV), "Ministerio de Educación y Ciencia de España" (PSL), "Departament de Física Teòrica de la Universitat Autònoma de Barcelona" (PSL), and "Conselho Nacional de Pesquisas do Brasil” (PSL).

\section{APPENDIX: EXTENSION OF THE BZSGT}

In this Appendix we present an extension of the BZSGT for the use of Hermitian matrices. This extension is already known for the elliptic case ${ }^{26}$ [SU (2) case]. Since the hyperbolic case can be treated in a completely similar way, we shall give only the results.

The $n$-soliton solution constructed from the seed solution $Q_{0}$ is

$$
\left(Q_{n}\right)_{a b}=\prod_{k=1}^{n}\left|\frac{\mu_{k}}{t}\right|\left(\left(Q_{0}\right)_{a b}-\sum_{k, l=1}^{n} \frac{\bar{N}_{a}^{(l)}\left(\Gamma^{-1}\right)_{l k} N_{b}^{(k)}}{\mu_{k} \bar{\mu}_{l}}\right)
$$

where, now the indices $a$ and $b$ take the values 1 and 2 , and

$$
\begin{aligned}
& \Gamma_{k l} \equiv \frac{m_{a}^{(k)}\left(Q_{0}\right)_{a b} \bar{m}_{b}^{l}}{\mu_{k} \bar{\mu}_{l}-t^{(2)}}=\bar{\Gamma}_{l k}, \\
& N_{a}^{(k)} \equiv m_{b}^{(k)}\left(Q_{0}\right)_{a b}, \\
& m_{a}^{(k)} \equiv m_{0 b}^{(k)} M_{b a}^{(k)}, \\
& \left.M^{(k)} \equiv \psi_{0}^{-1}\right|_{\lambda=\mu_{k}}, \\
& \mu_{k}=\alpha_{k}-r \pm\left[\left(\alpha_{k}-r\right)^{2}-t^{2}\right]^{1 / 2} .
\end{aligned}
$$

The bar denotes complex conjugation, $m_{0}^{(k)}$ and $\alpha_{k}$ are sets of complex constants. Here $\psi_{0}=\psi_{0}(t, r, \lambda)$ is the solution to the equations

$$
\begin{aligned}
& D_{t} \psi_{0}=\left(\left(t U_{0}+\lambda V_{0}\right) /\left(t^{2}-\lambda^{2}\right)\right) \psi_{0}, \\
& D_{r} \psi_{0}=\left(\left(t V_{0}+\lambda U_{0}\right) /\left(t^{2}-\lambda^{2}\right)\right) \psi_{0},
\end{aligned}
$$

where

$$
\begin{aligned}
& D_{t} \equiv \partial_{t}+\left(2 \lambda t /\left(t^{2}-\lambda^{2}\right)\right) \partial_{\lambda}, \\
& D_{r} \equiv \partial_{r}+\left(2 \lambda^{2} /\left(t^{2}-\lambda^{2}\right)\right) \partial_{\lambda}, \\
& U \equiv t Q_{0, t} Q_{0}^{-1}, \quad V=t Q_{0, r} Q_{0}^{-1} .
\end{aligned}
$$

The coefficient $\omega$ can be explicitly computed, we find 


$$
\begin{aligned}
\omega_{m}= & \hat{\omega}_{0}+2 \ln \left\{t-m^{2 / 2}\left(\prod_{k=1}^{m}\left|\mu_{k}\right|^{m+1}\right)\right. \\
& \times\left(\prod_{k, l}\left(\left|\mu_{k}-\mu_{l}\right|\left|\mu_{k}-\bar{\mu}_{l}\right|\right)^{-1}\right) \\
& \left.\times\left(\prod_{k=1}^{m} \frac{\left|\mu_{k}\right|^{2}-t^{2}}{\left|\mu_{k}^{2}-t^{2}\right|}\right)^{1 / 2} \operatorname{det} \Gamma\right\},
\end{aligned}
$$

where $\hat{\omega}_{0}$ denotes the $\omega$ function of the seed solution (metric and matter).

For the diagonal seed (5.2) Eqs. (A7) and (A8) can be solved along the pole trajectories, we $\operatorname{get}^{27}$

$$
\left.\psi_{0}\right|_{\lambda=\mu_{k}}=\left(2 \alpha_{k} \mu_{k}\right)^{1 / 2}\left(\begin{array}{cc}
\exp \left(-F_{k}\right) & 0 \\
0 & \exp F_{k}
\end{array}\right),
$$

where

$$
\begin{aligned}
F_{k} \equiv & \int \frac{t}{2 \mu_{k}}\left(\left(\mu_{k, t} \Lambda_{, t}+\mu_{k, r} \Lambda_{, r}\right) d t\right. \\
& \left.+\left(\mu_{k, t} \Lambda_{, r}+\mu_{k, r} \Lambda_{, t}\right) d r\right)
\end{aligned}
$$

The existence of $F_{k}$ is a consequence of (5.7) and that $\ln \mu_{k}$ satisfies the same Eq. (5.7).

For the diagonal seed solution case $^{27}$ the expressions (A2)-(A5) take the simple form,

$$
\begin{aligned}
& m_{1}^{(k)}=p_{k}\left(\mu_{k}\right)^{-1 / 2} \exp F_{k}, \\
& m_{2}^{(k)}=q_{k}\left(\mu_{k}\right)^{-1 / 2} \exp \left(-F_{k}\right), \\
& N_{1}^{(k)}=p_{k} t\left(\mu_{k}\right)^{-1 / 2} \exp \left(F_{k}-\Lambda\right), \\
& N_{2}^{(k)}=q_{k} t\left(\mu_{k}\right)^{-1 / 2} \exp \left(-\left(F_{k}-\Lambda\right)\right),
\end{aligned}
$$

$$
\Gamma_{k l}=\frac{t\left[p_{k} \bar{p}_{k} \exp \left(F_{k}+\bar{F}_{l}-\Lambda\right)+q_{k} \bar{q}_{k} \exp \left(-\left(F_{k}+\bar{F}_{l}-\Lambda\right)\right)\right]}{\left(\mu_{k} \bar{\mu}_{l}-t^{2}\right)\left(\mu_{k} \bar{\mu}_{l}\right)^{1 / 2}},
$$

where

$$
\begin{aligned}
& q_{k} \equiv m_{01}^{(k)} /\left(2 \alpha_{k}\right)^{1 / 2}, \\
& q_{k} \equiv m_{02}^{(k)} /\left(2 \alpha_{k}\right)^{1 / 2} .
\end{aligned}
$$

Note that the usual BZSGT relations, valid for real as well as for complex poles are obtained by letting $\bar{\mu}_{k} \rightarrow \mu_{k}$, $\bar{m}_{0 b}^{(k)} \rightarrow m_{0 b}^{(k)}, \bar{F}_{l} \rightarrow F_{l}, p_{k} \rightarrow \bar{p}_{k}$, and $q_{k} \rightarrow \bar{q}_{k}$ in (A1), (A2), (A12), and (A19).

'A. Lichnerowicz, Relativistic Hydrodynamics and Magnetohydrodyamics (Benjamin, New York, 1967).

${ }^{2}$ P. S. Letelier, Phys. Rev. D 22, 807 (1980).

${ }^{3}$ P. S. Letelier and R. Machado, J. Math. Phys. 22, 827 (1981); P. S. Letelier, Nuovo Cimento B 69, 145 (1982).

${ }^{4}$ P. S. Letelier, Phys. Rev. D 26, 2623 (1982).

${ }^{5}$ P. S. Letelier and P. S. C. Alencar, Phys. Rev. D 34, 343 (1986).

'S. S. Bayin, Phys. Rev. D 26, 1262 (1982); Astrophys. J. 303, 101 (1986).

${ }^{7}$ M. S. Madsen and G. Bailler, Astrophys. Space Sci. 114, 203 (1985).

${ }^{8}$ L. Herrera, G. J. Ruggeri, and L. Witten, Astrophys. J. 234, 1094 (1979)

M. Cosenza, L. Herrera, M. Esculpi, and L. Witten, Phys. Rev. D 25, 2527 (1982).

${ }^{9}$ D. W. Olson and J. Silk, Astrophys. J. 233, 395 (1979).

${ }^{10}$ P. J. E. Peebles, Astrophys. J. 257, 438 (1982)

${ }^{1}$ M. A. Hausman, D. W. Olson, and B. D. Roth, Astrophys. J. 270, 351 (1983).

${ }^{12}$ C. N. Yang, Phys. Rev. Lett. 38, 1377 (1977).

${ }^{13}$ V. A. Belinsky and V. E. Zakharov, Zh. Eksp. Teor. Fiz. 75, 1955 (1978);

77, 3 (1979) [Sov. Phys. JETP 48, 985 (1978); 50, 1 (1979)]

${ }^{14}$ R. Tabensky and A. H. Taub, Commun. Math. Phys. 29, 61 (1973).
${ }^{15}$ P. S. Letelier and R. Tabensky, Nuovo Cimento B 28, 407 (1975); P. S. Letelier, J. Math. Phys. 16, 1488 (1975).

${ }^{16} \mathrm{~L}$. Witten, Phys. Rev. D 19, 718 (1979).

${ }^{17} \mathrm{~J}$. Wainwright, W. C. W. Ince, and B. J. Marshman, Gen. Relativ. Gravit. 10, 259 (1979); P. S. Letelier, J. Math. Phys. 20, 2078 (1979).

${ }^{18}$ See, for instance, J. L. Synge, Relativity: The General Theory (North-Holland, Amsterdam, 1966).

${ }^{19}$ B. J. Carr and E. Verdaguer, Phys. Rev. D 28, 2995 (1983).

${ }^{20} \mathrm{~J}$. Ibañez and E. Verdaguer, Phys. Rev. Lett. 51, 1313 (1983).

${ }^{21} J$. Ibañez and E. Verdaguer, Phys. Rev. D 31, 251 (1985).

${ }^{22} \mathrm{~J}$. Ibañez and E. Verdaguer, Astrophys. J. 306, 401 (1986).

${ }^{23} \mathrm{E}$. Verdaguer, in Observational Theoretical Aspects of Relativistic Astrophysics and Cosmology, edited by J. L. Sanz and L. J. Goicoechea (World Scientific, Singapore, 1985).

${ }^{24} \mathrm{X}$. Fustero and E. Verdaguer, Gen. Relativ. Gravit. 18, 1141 (1986)

${ }^{25}$ E. P. Liang, Astrophys. J. 204, 235 (1976).

${ }^{26}$ P. S. Letelier, J. Math. Phys. 23, 1175 (1982); 27, 615 (1986).

${ }^{27}$ P. S. Letelier, J. Math. Phys. 25, 2675 (1984); 26, 467 (1985); 27564 (1986). 